spectromicroscopy, photo-electron microscopy and holography, to crystallography, biology, medicine, and three-dimensional microstructure fabrication.

Much of the discussion concentrated on the expected benefits of high brightness, which is identical to spectral brilliance, i.e., to the number of photons per time and unit area into a unit solid angle for a given frequency or wavelength interval. An obvious advantage is the possibility of achieving high fluxes on small samples. This, in general, means a very good signal-to-noise ratio with the result that extremely short measuring or exposure times are sufficient for obtaining the desired data. This feature ensures that measurements may be done under stable conditions, although controlling possible radiation damage must be seriously considered. Short measuring times allow one to study the effect of varying experimental conditions such as the specimen temperature or applied pressure very quickly.

Other possibilities include the time-resolved observation of the folding of proteins and of cellular dynamics by monitoring the flow of fluorescent chemicals (hence the great potential for high-brilliance synchrotron radiation sources in biology, biochemistry and medicine). High flux on small samples also clearly favours measurements on exotic materials that are not available in large quantities, of matter under very high pressure, and the identification and subsequent spectroscopic investigation of components in nanoscale structures.

Precautions will in general have to be taken to limit radiation damage or excessive heating of specimens. High-intensity sources may also saturate existing detectors so their availability will encourage the development of detector technology. High brightness must also be accompanied by exceptional beamline optics in order to preserve the phase
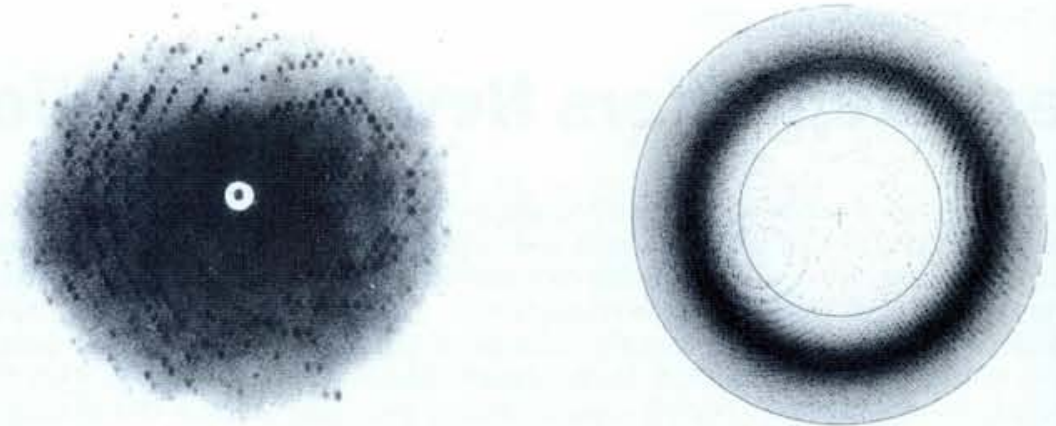

The X-ray Laue pattern (on the left) of hemoglobin ( $6.45 \times 10^{4} \mathrm{~K}$ Daltons) as obtained with a sealed-tube X-ray source (courtesy of M. Perutz, Cambridge), compared with the pattern for a bovine enterovirus ( $>10^{7}$ Daltons) obtained using synchrotron radiation at the Daresbury facility (courtesy of D. Stuart, Oxford).

space up to the sample positions. Problems of this type are best solved if the layout of the sources and the planning of experiments are done in cooperation at an early stage.

\section{Two Examples}

The advantage of high photon fluxes is well illustrated by comparing diffraction patterns of complicated structures obtained employing conventional $\mathrm{X}$-ray sources and synchrotron radiation (see figure). The drastic enhancement of well-resolved information obtained using modern instrumentation is truly impressive.

Another important example concerns the surface-sensitive method of photo-electron spectroscopy. Using a conventional rotating anode source to record a valence-band spectrum of a solid, typically using a scan of $10 \mathrm{eV}$ with a resolution of $0.3 \mathrm{eV}$ and good statistics, needs a few hours. This poses severe contamination problems for many materials. However, the same spectrum may be obtained from a bending-magnet source in a few minutes. Undulator sources deliver yet an- other factor of $10^{5}$-times more radiation intensity, implying that the same energy range of electronic states may be scanned within a fraction of a second to produce spectra with a much improved energy resolution from a significantly smaller spot on the sample. One can, in fact, obtain two-dimensional photoelectron "images" with a resolution that may reach 100-200 A. Additional information can be obtained by monitoring the spin dependence of the emission, where the inherently low efficiency of detection methods makes high-intensity sources particularly useful.

The workshop concluded that the SLS project is timely as it would ensure world-wide leadership for the foreseeable future in photon production at energies between $10 \mathrm{eV}$ and $3 \mathrm{keV}$, complementary to other, powerful sources operating at even higher energies. It would also make a respectable contribution to the large and still growing international community of scientists from many differen disciplines who use synchrotron light sources.

H.R. Ott, ETH, Zurich

\title{
Teachers in National Physical Societies
}

Summary of a survey carried out by the Forum for Education (questionnaire sent in January 1994; 16 countries replied formally)

\begin{tabular}{|c|c|c|c|c|c|c|c|c|c|c|c|c|c|c|c|c|}
\hline \multicolumn{2}{|r|}{ Society } & \multirow{2}{*}{$\frac{\text { Div }}{y}$} & \multicolumn{2}{|c|}{ Union Orgs } & \multirow{2}{*}{ Conf } & Gen & \multirow{2}{*}{$\begin{array}{l}\text { Act } \\
c\end{array}$} & \multirow{2}{*}{$\begin{array}{l}\text { Govt } \\
\text { advice }\end{array}$} & \multirow{2}{*}{$\begin{array}{l}\text { Advice } \\
\text { curricula }\end{array}$} & \multirow{2}{*}{$\begin{array}{l}\text { Comp } \\
-/ y / y\end{array}$} & \multirow{2}{*}{$\begin{array}{l}\text { Exch } \\
-1-\end{array}$} & \multirow{2}{*}{$\begin{array}{l}\text { Teach bu } \\
4 / 30\end{array}$} & Soc bu & \multirow{2}{*}{ Links } & \multicolumn{2}{|c|}{ Prizes Int } \\
\hline A & $1230 / \mathrm{NR} / 100$ & & $y$ & $y /-1-1-$ & & $y / 1$ & & & & & & & $/ 4 / 12$ & & $y$ & $y$ \\
\hline B & $400 / 5 / 100$ & y & $y$ & y $1-1-1$. & - & sat /1 & - & - & - & $-|y|-$ & $-1-$ & $-1=$ & $/ 12 / \quad 16$ & - / c; lab visits / - & - & . \\
\hline $\mathrm{CH}$ & $1430 / N R / 100$ & : & : & y $/-1-1=$ & - & -10.5 & 5- & - & $\mathrm{n}$ & -1.1 & $.1-$ & -1. & $1-1$. & $\mathrm{c} / \mathrm{-}$ & - & - \\
\hline CR & $190 / 60 / 100$ & $y$ & $y$ & $-|y|-\mid$ & - & - & sem & plan & - & $-|y|-$ & $-1-$ & -1. & $\mathrm{col} / ? / ?$ & $\mathrm{c} / \mathrm{-}$ & - & - \\
\hline$E$ & $3000 /<1 / 100$ & y & ? & $-1-1-1=$ & $?$ & 12 & $?$ & $?$ & $?$ & $y /-1$. & -1. & -1. & i;a / $4 / 80$ & $-1-$ & $?$ & $?$ \\
\hline EE & $230 / 7 / 100$ & comm & - & $y / y /-1$. & - & $y / 1$ & - & - & - & $-|y|=$ & -1. & $=1$. & $-1.1=$ & $\mathrm{c} /$ & - & - \\
\hline$F$ & $2480 /<1 /<100$ & comm & - & $y / y /-1-$ & - & y 12 & - & - & teaching & $y / . /=$ & $-1-$ & $\cdot 1-$ & $|5| 36$ & staff / - & - & - \\
\hline FIN & $1000 / 20 / 100$ & $y$ & - & $y / y / y / y$ & - & y $/ 1$ & c & - & - & $-1-1-$ & $-1-$ & $-1-$ & a / $/ 4 / 100$ & dep; c / - & - & - \\
\hline G & $27050 / 2 / 100$ & $y$ & $y$ & $y / y /-/ y$ & 1 & $/ 1$ & s & - & - & $-|y|-$ & -1 & -1. & i $/ 10 / 110$ & $\mathrm{c} / \mathrm{l}$ & - & $y$ \\
\hline $\mathrm{H}$ & $1490 / 43 / 100$ & $y(2)$ & - & $-1-1.1$. & - & 13 & - & react & - & $y / y / y$ & $y /-$ & $12 / ?$ & $\mathrm{col} / 12 / 40$ & $-1 \mathrm{~s}$ & - & 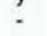 \\
\hline 1 & $1350 / 2 / 100$ & comm & $y$ & $y /-|y|-$ & - & $y / 1$ & sem & react & curricula & $-1-1=$ & $-1-$ & $-1=$ & i $/ 4 / 80$ & $\mathrm{c} / \mathrm{y}$ & $y$ & - \\
\hline $\mathrm{N}$ & $850 / 30 / 100$ & $y$ & $y$ & $y /-1-1$ & 3 & y $/ 1$ & - & - & - & $-/ y / y$ & -1 & $-1=$ & si $/ 4 / 20$ & $\mathrm{c} / \mathrm{-}$ & - & - \\
\hline NL & $3600 / 5 / 100$ & comm & - & $y / y /-1-$ & 1 & -11 & sem; c & :- & - & $y / y /-$ & $-1-$ & -1. & a $/ 26 / 12$ & c; dep; lect; tut / - & grants & $y$ \\
\hline $\mathrm{P}$ & $1700 / 67 / 100$ & y & - & -1.1 .1 & - & sat/2 & sem & - & - & $-|y|-$ & $-1 / y$ & -1. & a $/ 4 / 80$ & $\mathrm{EU} / \mathrm{-}$ & - & : \\
\hline $\mathrm{PL}$ & $1800 / 13 / 25$ & $y$ & - & y $1-1-1-$ & ? & -12 & $?$ & $?$ & $?$ & $-|y|-$ & $-1-$ & $6 / 20$ & a / 6/100 & -1 & ? & $?$ \\
\hline $\mathrm{R}$ & $1200 / 4 / 100$ & $y$ & - & $-1-1-1=$ & - & 12 & sem & - & ed reform & $y / y /=$ & $.1-$ & -1. & $0.1 / 4 / 30$ & -1 & - & - \\
\hline $\mathrm{S}$ & $1050 / 25 / 100$ & $y$ & $y$ & $y /-1-1=$ & ? & 12 & - & $y$ & & $-|y|=$ & $-1-$ & -1. & i / $4 / 30$ & res; c; lect / - & - & $y$ \\
\hline & $260 / 50 / 100$ & y & - & $y / y /-1$ & : & $/ 1$ & lect & - & teaching & $y /-1-$ & -1. & -1. & $\mathrm{i} / 6 / 20$ & $\mathrm{c} / \mathrm{l}$ & - & . \\
\hline UK & $18500 / 10 / 100$ & $y$ & $y$ & $y / y /-/ y$ & 1 & $/ 1$ & c & react & various & $y / y /$. & $-1-$ & $6 / 40$ & $a ; i / 12 / 80$ & -1. & $y$ & - \\
\hline
\end{tabular}

Society: nat. soc. (NS) membership / \% teachers (NR = not recorded) $/ \%$ of fee

Div: $\quad$ organized as a Division (comm = national committee only)

Union: teachers' union exists Orgs: other teacher organizations exit / collaborate / joint members / joint activities

Conf: annual teachers conference (frequency in years) Gen: annual (or similar) general conference involving education / frequency in years

Act: $\quad$ other activities organized for teachers (courses; schools; seminars; lectures)

Govt: advise government Anal: survey, analysis, etc. carried out (subject/s)

Comp: national competition / Physics Olympiad participation / training courses for Olympiad

Exch: exchange teachers between conferences / between countries

Teach bull: teachers bulletin published: no. issues p.a. / pages per issue

Soc bull: society bulletin published: contents relevant to teachers (articles; information; special issues; coloumn; \%) / no. issues p.a. / pages per issue

Links: teachers with universities (courses, university staff teach; special univ. departments; teachers act as tutors; resource centre; lectures for

Prizes: teachers / teachers with institutes (scholarships)

216 Europhys. News 25 (1994)

Int: participation in international bodies (IUPAP, Int. Baccalaureate, EU, OECD, etc.) 